\title{
Diversidad del género Selaginella P. Beauv (Selaginellaceae) en el departamento del Chocó
}

\section{Diversity of Selaginella P. Beauv (Selaginellaceae) in the department of Chocó}

\author{
Leider Palacios-Palacios*
}

\section{RESUMEN}

El género Selaginella, perteneciente a la familia Selaginellaceae cuenta con casi 700 especies distribuidas sobre todo en zonas tropicales y subtropicales. En el Chocó biogeográfico cuenta con aproximadamente 38 especies. Con el objetivo de conocer la diversidad de este taxa en el departamento de Chocó, se estudiaron las colecciones del Herbario CHOCÓ, a través de su confrontación con claves especializadas para el género, revisión de literatura y dos salidas al Corregimiento de Pacurita, Municipio de Quibdó, donde se colectaron al azar Selaginella en sitios como bordes de quebradas y caminos, asociadas con sustratos como hojarascas, tronco, rocas y suelo. Reportando doce especies en colecciones del Herbario CHOCÓ y a manera de las salidas de campo se incluyen Selaginella rupestris y $S$. willdenowii a la lista de plantas del departamento, así como registros fotográficos, descripciones sucintas y sinónimos de las especies en conocimiento.

Palabras clave: Chocó biogeográfico; Pacurita; Selaginella;

Selaginellaceae.

\section{ABSTRACT}

The genera Selaginella, belonging to the family Selaginellaceae has nearly 700 species distributed mainly in tropical and subtropical areas. In the Choco has approximately 38 species. In order to know the diversity of this taxa in the Chocó, we studied the collections of the Herbarium-CHOCÓ, through its confrontation with specialized keys for the genre, literature review and two trips to Corregimiento Pacurita, district of Quibdo, where they were collected randomly at sites such as Selaginella the edges of streams and roads, associated with substrates such as leaves, trunks, rocks and soil. Reporting twelve species in the herbarium collections and CHOCO way to the field trips are included Selaginella rupestris and S. willdenowii to the list of plants of the department, as well as photographic records, brief descriptions and synonyms of species in knowledge.

Keywords: Chocó Biogeográfico; Pacurita; Selaginella; Selaginellaceae.

\section{INTRODUCCIÓN}

Los Pteridofitos en Colombia se encuentran representados por $\sim 1600$ especies, las cuales están ampliamente distribuidos en todo el país (Méndez et al. 2002). En el Chocó
* Investigador, Grupo de Biosistemática, Universidad Tecnológica del Chocó «Diego Luis Córdoba». e-mail: leipapal@yahoo.com leipal@hotmail.com

Recibido: agosto 12, 2008 Aceptado: agosto 26, 2008 
dad sobresale el género Selaginella, perteneciente a la familia Selaginellaceae que cuenta con casi 750 especies distribuidas sobre todo en zonas tropicales, crecen en lugares cálidos y húmedos aunque existen algunas especies en desiertos y en zonas de alta montaña (Prada 2004). El Chocó biogeográfico cuenta con aproximadamente 38 especies y en el departamento del Chocó 24 (Forero \& Gentry 1989; Murillo-A 2004). Con el objetivo de contribuir al estudio taxonómico de este taxa, el presente trabajo da a conocer un registro sucinto de algunas especies del género Selaginella presentes en el Herbario CHOCÓ, como un aporte al estudio y conocimiento de este grupo botánico en particular y de la familia Selaginellaceae en general para el Chocó.

\section{MATERIALES Y MÉTODOS}

Se estudiaron las colecciones del Herbario CHOCÓ que fueron confrontadas e identificadas teniendo en cuenta las publicaciones de Alston(1955), Alston etal. (1981), Gregory et al. (1979), Jermy (1986) y Valdespino (1993), la literatura especializada de Triana-M \& Murillo-A. (2005), Méndez et al. (2002), Rodríguez (2002) y Baker (1883); su posterior comparación con las colecciones de imágenes tipo presentes en las bases de datos del Instituto de Ciencias Naturales (ICN) www.unal.edu.co/icn/, el Jardín Botánico de Missouri (MO) www.tropicos.org/Name Speci mens.asp, el New York Botanicals Garden (NY) www.sciweb.nybg.org/Virtual Herbarium.asp, el Real Jardín Botánico (KEW) www.kew.org/ data/index.html y la clave para las especies del género por M.E. Fraile, P. Somers, Jr. y R.C. Moran. El trabajo se complementó con dos salidas al corregimiento de Pacurita que dista 7 km vía carreteable de su cabecera municipal Quibdó, ubicado geográficamente a $5^{\circ} 41^{\prime}$ de latitud norte y $76^{\circ} 40^{\prime}$ de longitud oeste a $53 \mathrm{~m}$ de altura. Esta zona presenta una humedad relativa de $87 \%$, una temperatura promedio de $26^{\circ} \mathrm{C}$ yuna precipitación anual de $7.776 \mathrm{~mm}$ (CODECHOCÓ 1997), y pertenece según Espinal (1977) a la formación de bosque pluvial tropical (bp-T). En sitios como bordes de quebradas y caminos e indicando el tipo de sustrato en el cual se encontrasen asociadas (hojarascas, tronco, rocas, suelo, entre otros) se colectaron al azar las Selaginellas de acuerdo con sus características morfológicas de dos a tres ejemplares de cada morfoespecie observada.

\section{RESULTADOSYDISCUSIÓN}

Se registraron doce especies en colecciones del Herbario CHOCÓ y a manera de las salidas de campo se incluyen como novedades Selaginella rupestris y $S$. willdenowii a la lista de plantas del departamento (Tabla 1), así como registros fotográficos, descripciones sucintas y sinónimos de las especies en conocimiento.

\section{TRATAMIENTOTAXONÓMICO}

Selaginella Palisot de Beauvois. in Mag. Encyclop.(Paris), 9(5):478(1804). 1804

Tipo: Selaginella spinosa P. Beauv. - Prodrome des Cinquième et Sixième Familles de l'Aethéogamie 112. 1805.

Las selaginellas pertenecen a la división Lycophyta, junto con los Lycopodium (Licopodios) e Isoetes. Estos pueden poseer especies erguidas, rastreras o colgantes, tallos, raíces y hojas verdaderas, pero no ocupan un lugar tan influyente en la flora actual, debido tal vez a que no han evolucionado, pero sus estrategias reproductivas en el perfeccionamiento del esporofilo, con la diferenciación del aparato vegetativo han favorecido su permanencia y colonización de muchos hábitats en la actualidad (Prada 2004).

Las especies del género Selaginella, se caracterizan por ser plantas terrestres, rupícolas y ocasionalmente epifitas, tallos articulados o no articulados, raíces en su mayoría adventicias y dicótomas, localizadas en los extremos de los rizóforos originados en la superficie ventral o dorsal de las bifurcaciones del tallo, hojas isofilas arregladas en cuatro hileras, dos hojas medias en la superficie dorsal y dos laterales, con una axilar ventral en cada dicotomía del tallo; estróbilos al final de las ramas terminales, compuesto por esporofilos bien diferenciados; megasporangios en la parte basal del estróbilo, frecuentemente con 4 megásporas; microsporangios en la parte distal del estróbilo, con numerosas microsporas; $\mathrm{x}=8,9,10,12$. (Apoyado en: Alston et al. 1981, Alston 1955; Gregory et al. 1979; Jermy 1986; Valdespino 1993). En cuanto a los hábitats, según registros de colecciones y salida de campo, se observó que por lo general crecen en lugares húmedos y sombreados, donde los sustratos más apreciados para el crecimiento de estas especies son la hojarasca y tronco-roca; el hábito de crecimiento de las especies estuvo variando entre epífitas, terrestres y humícolas.

El principal uso referenciado para las selaginellas es ornamental, además de servir a nivel ecológico en la reserva de agua y cubierta del suelo o en ocasiones sirviendo de sustrato en los troncos para que germinen las semillas de otras especies epífitas (orquídeas, aráceas, ciclantáceas) u otros helechos, etc.

Selaginella articulata (Kunze) Spring. In Mart. Flora Brasilensis 21: 182.1838.

Tipo: Peru, Poeppig s.n.(Holótipo)(Bra.).

=Lycopodium articulatum Kunze, Linnaea 9: 10 . $1834[1835]$.

Tallos pelosos en el envés, ramificados en su extensión, generalmente más oscuros que las hojas, con ramificación dicotómica; hojas axilares elípticas, base con aurícula corta; hojas laterales asimétricas, márgenes enteros, ápice obtuso; hojas medias asimétricas, márgenes entero, ápice acuminados. 


\section{Tabla 1 \\ Listado y distribución de especies del género Selaginella en análisis de sus colecciones en el Herbario CHOCó}

Listado de especies
Área fitogeográfica Forero \& Gentry (1989)

Altura (msnm)

$\begin{array}{lll}\text { Selaginella articulata (Kunze) Spring } & \text { RCP, SPC, SHN } & 50-200 \\ \text { Selaginella atirrensis Hieron. } & \text { RRSJ, SPC, TADCA } & 50-150 \\ \text { Selaginella exaltata (Kunze) Spring } & \text { RRSJ, SPC } & 150-200 \\ \text { Selaginella geniculata (C. Presl) Spring } & \text { RU, RCP, SPC } & 0-480 \\ \text { Selaginella haematodes (Kunze) Spring } & \text { RU, RRSJ, SPC } & 0-270 \\ \text { Selaginella horizontalis (C. Presl) Spring } & \text { SPC } & 0-100 \\ \text { Selaginella kunzeana A_Braun } & \text { TADCA, SPC } & 0-200 \\ \text { Selaginella longissima Baker } & \text { TADCA, RCP, RRSJ } & 10-1200 \\ \text { Selaginella oaxacana Spring } & \text { RRSJ, SPC } & 100-250 \\ \text { Selaginella rosea Alston } & \text { Límite Chocó-Antioquia } & 1500-2280 \\ \text { Selaginella rupestris (L_) Spring } & \text { SPC } & 40 \\ \text { Selaginella speciosa A_Braun } & \text { SPC, RRSJ } & 80-200 \\ \text { Selaginella tomentosa Spring } & \text { SPC, RRSJ } & 150-500 \\ \text { Selaginella willdenowii (Desv_ex Poir_) Baker } & \text { SPC } & 50\end{array}$

Región de Urabá = RU; Serranía del Darién = SD; Selva Húmeda del Norte = SHN; Región de la Costa Pacífica = RCP; Tierras Altas del Carmen de Atrato = TADCA; Selva Pluvial Central = SPC; Región de San José del Palmar = RSJP; Región del Río San Juan = RRSJ .

Observaciones: Similar en tallos pelosos con S. exaltata, pero ésta es mucho más grande (1-3 m) y con ramificación pinnada. Presente en Honduras, Panamá, Colombia (Antioquia, Chocó), Ecuador y Perú. Desde los 0-1750 m (Missouri Botanical Garden - $w^{3}$ - Specimen Data Base - 2007).

Material examinado: COLOMBIA.Chocó; Nuqui: Cabo Corrientes, Corr. Arusí, Estación Ecológica el Amargal, $50 \mathrm{~m}$, Suárez, 587 Quibdó: Corr. Tutunendó, 100 m, Santa, 1141. Riosucio: Corr. Macondo, Cerro El Cuchillo, 50-200 m, Cardenas, D.1500, 1935.

Selaginella atirrensis Hieron. In Engl. et Prantl. Nat. Pflanzenfam 1(4):711.1901.

Tipo: Costa Rica: Cartago: Atirro, 2000 ft. J.D. Smith 5103, (Lectotipo) (B).

=Selaginella intacta autc., non Baker

Tallos de aproximadamente $1 \mathrm{~mm}$, bistelicos, glabros, prostrada, largamente rastrera, con rizóforos en toda su longitud; hojas medias desiguales, al igual que las laterales, hojas axilares ovadas a lanceoladas ciliadas, de ápice agudo.

Observaciones: A menudo postradas sobre rocas húmedas, arena. Con presencia en los países de Costa Rica, Panamá, Colombia (Antioquia, Chocó), Ecuador, Perú. Desde los 0-2400m. (Missouri Botanical Garden - $w^{3}$ - Specimen Data Base - 2007).
Material examinado: COLOMBIA. Chocó; Itsmina: Andagoya, 60 m, Kiliip, E.P. 5379, R. San Juan, Killip, E.P 35379. Quibdó: Archer, W.A. 1666, carr. Quibdó-Tutunendó, km 18, 300 m, Santa, J. 417; carr. Tutunendó-San Francisco de Ichó, R. Ichó, 50 m, Santa, J. 1154 1167. Bagado: Certegui, 130-150m, Juncosa, A. 1502.

Selaginella exaltata (Kunze) Spring. Bull. Mem. Acad. Roy. Sci.Bruxelles 10 (1):234. 1843.

Tipo: Peru, Poeppig 1953(LG)

= Lycopodioides exaltata (Kunze) Kuntze; Rev. Gen. Pl. 1:826(1891)

=Lycopodium exaltatum Kunze; Linnaea 9:8(1834)[1835]

= Selaginella strobilifera $\mathrm{H}$. Christ; Bull. Herb. Boissier, ser. 2, 2, 1:72(1901)

Fácil de reconocer por su tamaño, largas ramas, pinnadas; hojas axilares ausentes o semejantes con las hojas medias; hojas laterales en la base de las ramas, oblongas, adnadas en la base o decurrentes en la base, márgenes enteros, ápices acuminados.

Observaciones: Presente en bordes de caminos, barrancos, zonas abiertas. Com registros en Costa Rica, Panamá, Colombia (Amazonas, Antioquia y Chocó), Ecuador, Perú, Bolívia y Brasil. Desde 0-700 m(Missouri Botanical Garden$w^{3}$ - Specimen Data Base - 2007). 
Material examinado: COLOMBIA. Chocó; Itsmina: Andagoya, R. San Juan, campamento Compañia Mineros del Chocó, 60 m, Forero, E 5189. Paimadó: can. Panamericana, R. SanPablo, R. Pató, 150-200m, Forero, E 5535. Quibdó: can. Tutunendó-San Francisco de Ichó, R. Ichó, 50 m, Santa, 1162.

Selaginella geniculata (C. Presl) Spring. Bull. Mem. Acad.Roy. Sci.Bruxelles 10(1):230. 1843.

Tipo: Ecuador o Perú, Haenke s.n. (Holótipo) (PR)

= Lycopodium geniculatum C. Presl Reliquiae Haenkeanae 1(1): 80. 1825.

=Lycopodioides geniculata (Presl)Kuntze; Rev. Gen. Pl. 1:826(1891)

= Lycopodium flabellatum Kunze; Linnaea 9: 9 (1835)

[non L., 1753

= Selaginella conduplicata Spring; Fl. Bras. 1(2): 129 (1840)

= Selaginella elongata Klotzsch; Linnaea 18: 522 (1844)

= Selaginella ferruminata Spring; Bull. Acad. Roy. Sci. Bruxelles 10:231 (1843)

= Selaginella nodosa Presl; Bot. Bem., 150 (1844)

Tallos principalmente erectos, bistélicos, glabros, nudos hinchados y hojas laterales asimétricas con aurículas deltadas a cuadradas; hojas axilares elípticas, márgenes enteros, ápices obtusos a agudos; hojas medias asimétricas con ápice agudo.

Observaciones: Especie presente en los países de Panamá, Colombia (Antioquia, Chocó, Risaralday Valle del Cauca), Ecuador y Perú. Desde los 0-1900m (Missouri Botanical Garden - $w^{3}$ - Specimen Data Base - 2007).

Material examinado: COLOMBIA. Chocó; Acandi: Snía. del Darién, Mpio., Juncosa, A. 658, 615. Bahía Solano: Corr. El Valle, Trocha Ensenada de Utría, Espina, J., F. García., S. Pino 2679. Quibdó: Carmen de Atrato, carr. Quibdó-El Diecisiete, 350 m, Santa, J. 1138; Corr. Guayabal, R. Hugón, García, L. 072; km 159, elevación 480 m. Croat, T.B. 55934.

Selaginella haematodes (Kunze) Spring. In Mart. Flora Brasiliensis 1(2): 126. 1840.

Tipo: Perú, Poeppig s.n. (K)

=Lycopodium haematodes Kunze Linnaea 9:9. 1834[1835].

=Lycopodioides haematodes (Kunze) Kuntze; Rev Gen Pl.1:826.(1891)

=Lycopodioides karsteniana (A. Br.) Kuntze; Rev. Gen. Pl. 1:826(1891)

= Selaginella filiciana Spring; Mem. Acad Royal Sci Belgique 24: 189. (1849)

= Selaginella karsteniana Klotzsch ex A. Br.; App Ind Sem Hort Berol. 18. (1857)

Planta terrestre, trepadora, tallo escamoso, pinnas pequeñas, erectos, coloridos de rojo brillante en algunas partes, no articulados; rizóforos solos en la base; hojas laterales ovadas a ovadas-oblongas, base redondeada, margen entero; hojas axilares ovadas, base redondeada, margen denticulado, ápice agudo; hojas medias ovado-lanceoladas, base cuneada, margen denticulado, ápice acuminado a aristado.

Observaciones: Presente en zonas húmedas, zonas inmudables, bosques secundarios. Presente en Panamá, Bolivia, Colombia (Antioquia, Chocó, Meta), Ecuador, Perú, Venezuela y en Trinidad, Zona Caribeña. Desde 0-300m (Missouri Botanical Garden - $w^{3}$ - Specimen Data Base 2007).

Material examinado: COLOMBIA. Chocó; Acandi: Snía. del Darién, E Mpio., Juncosa, A. 652. Itsmina: R. San Juan, Negría, 50 m, Killiip, E.P. 65029; Corr. Primera Mojarra, GIPN 420. Quibdó: Carmen de Atrato, Juncosa, A. 511; Corr. Bebará, La Calle, Río Bebará, Córdoba, W.A. 419.

Selaginella horizontalis (C.Presl) Spring. Bull Mem Acad Royal Sci Bruxelles 10 (1): 226. 1843.

Tipo: Perú, Haenke s.n. (Holótipo) (foto US ex PR)

=Lycopodium horizontale C. Presl Reliquiae Haenkeanae 1(1): 78.1825 .

Tallos de 1-2 mm de ancho, hojas medias pel-tadas, con una aurícula basal, cilios 0.5-1.5 mm y hábito de crecimiento postrado; rizóforos fuertemente a lo largo de los tallos; hojas axilares lanceoladas a elípticas, margen ciliado; hojas laterales asimétricas, margen ciliado y ápice agudo.

Observaciones: Presente en zonas urbanas, matorrales; en países como Costa Rica, Panamá, Bolivia, Colombia (Antioquia, Chocó), Ecuador, Perú y Venezuela. Desde 0$1200 \mathrm{~m}$. (Missouri Botanical Garden $-w^{3}$ - Specimen Data Base - 2007).

Material examinado: COLOMBIA. Chocó; Quibdó: 0m, Archer W, A. 1805; Barrio San Judas, Córdoba. W.A. 349; carr. Tutunendó, Km 14,90m, Gentry, Á. H. 24133; Corr. Tutunendó, 100 m, Santa, J. 1140.

Selaginella kunzeana A_Braun. Ann.Sci. Nat., Bot., ser. 5, 3:296. 1865.

Tipo: Perú, 1829, E.F. Poeppig s.n. (MO).

$=$ Lycopodium radiculosum Kunze ex A. Br.; Ann. Sci. Nat. V, 3:296 (in syn.) (1865)

= Selaginella poeppigiana sensu Kunze; Linnaea 9: 11 (1834)

Tallos bistélicos, glabros, rastreros; rizóforos a lo largo de todo el tallo; hojas axilares lanceoladas a elípticas, margen serrado y ápices agudos; hojas laterales asimétricas, margen acroscópico, serrados, ápices agudos; hojas medias asimétricas, base peltada, margen serrado a enteros, ápices acuminados a aristados.

Observaciones: Habitual en zonas intervenidas, húmedas. En los países de Costa Rica, México, Nicaragua, Panamá, Colombia(Antioquia, Chocó), Ecuadory Perú. Desde 0-200m. 


\section{Bioetnia Volumen 5 No 2 (julio-diciembre), 2008}

(Missouri Botanical Garden - $w^{3}$ - Specimen Data Base 2007).

Material examinado: COLOMBIA. Chocó; El Carmen: carr. Quibdó-Medellín, km 162, Santa, J. 178; can. TutunendóElCarmen, cnías. Campamento ElDoce, $60 \mathrm{~m}$, Forero, E. 5866. Quibdó: Barr. San Judas. Córdoba, W. A. y F. García 349. Tadó: Playa de Oro. Carr. Santa Cecilia. Espina, J. \& L. A. Arias1193, Espina, J. \& M. Mosquera 2398.

Selaginella longissima Baker.J. Bot. Londr. 19:208. 1881. Tipo: Colombia: Antioquia: $3000 \mathrm{ft}$ Kalbreyer 1815 (Holotipo) $(\mathrm{K})$.

= Lycopodioides longissima (Baker) Kuntze; Rev. Gen. Pl. 1:826(1891)

Planta de tallo terrestre a epifito, como enredadera, de aproximadamente $1 \mathrm{~mm}$; rizóforos en toda su longitud; hojas axilares lanceoladas, margen serrado, ápices agudos; hojas laterales oblongas-lanceoladas, margen ciliado; hojas medias peltadas, margen serrados, ápices acuminados.

Observaciones: Presente en zonas húmedas, intervenidas. En los países de Colombia (Chocó, Valle del Cauca) y Ecuador. Desde 10-1200m(Missouri Botanical Garden - $w^{3}$ Specimen Data Base - 2007).

Material examinado: COLOMBIA. Chocó; BagadóCertegui, 130-150m. Juncosa, A. 1504. Bahía Solano: Río Mecana, 5-10 m, Juncosa, A. 1673. Itsmina: Cerro. Primera Mojarra, GIPN 421; Raspadura, Unión Panamericana, Ledesma, E. et al. 366. Quibdó: Carr. Medellín, Tutunendó. Ca. 9 km de Quibdó, Croat, T. B. 46178.

Selaginella oaxacana Spring. Bull Mem Acad Royal Sci. Belgique 24: 177. 1849.

Tipo: México: hab. In sylvis humidis Chinantla, prov. Oaxaca Galeotti 6608 bis, (presumably LG; IT: P).

= Lycopodium flabellatum var. strictum Mart. \& Gal.; Mem Acad Royal Belgique 15: 12. 1842.

= Selaginella costaricensis Hieron. Engl \& Prantl, Nat Pfl. L (4): 683, (127). [1901] 1902

= Selaginella wendlandii Hieron. Engl \& Prantl Nat Pfl. L (4): 683, (126). [1901] 1902

Planta terrestre, tallos erectos, distales, isofilos, no articulados; rizóforos basales gruesos; hojas laterales ovadas a oblongas, base redondeada, margen entero, ápices agudos; hojas axilares ovadas a elipticas, base redondeada; hojas medias ovadas, margen ciliados, apice acuminado, aristados o acuminados.

Observaciones: Presentes en zonas intervenidas, húmedas, pastizales, regiones templadas. Para países como: Costa Rica, México, Panamá, Colombia (Antioquia, Chocó, Nariño) y Ecuador. Desde 0-1600m (Missouri Botanical Garden - $w^{3}$ Specimen Data Base - 2007).

Material examinado: COLOMBIA. Chocó; Bahía Sola- no: Puerto Mutis, 8 km., vía El Valle, 50 m, Lellinger D. B. 121. Itsmina: Espinal, J. etal. 1351; Mojarras de Tadó, $8.5 \mathrm{~km}$ del Mpio., 150-250 m, Lellinger, D.B 391. Quibdó: Qda. Concepción, O m, Archer, W. A. 1974

Selaginella rosea Alston. J Bot Br Foreign. London 70: 281.1932.

Tipo: Colombia. Forests of Huangubio, in highlands of Popayán. Alt. 1700-1800 m. F. C. Lehmann B.T. 845 (Isotype)

Planta terrestre, tallo erecto, glabro, de color rozado; rizóforos básales gruesos; hojas laterales lanceoladas a oblongas, base redondeada, cuneada, margen entero, ápices agudos; hojas axilares ovadas, base redondeada; hojas medias ovadas, margen ciliados, ápices acuminados.

Observaciones: Presente en zonas húmedas, de grandes alturas, intervenidas, sólo para Colombia en los departamentos de Antioquia y límites con el departamento del Chocó. Desde los 2140-2280 m (Missouri Botanical Garden - $w^{3}$ Specimen Data Base - 2007).

Material examinado: COLOMBIA. Chocó; Mpio. Salgar; límites entre Antioquia y Chocó, 0559'N07607'W. $15 \mathrm{~km} \mathrm{de}$ Doraudo, 2280 m, Zaraucchi, L. J. et al. 5965.

Selaginella rupestris (L_) Spring. Flora, oder (Allgemeine) Botanischer Zeitung. Regensberg, Jena 21: 149, 182. 1838.

Tipo: USA. New Mexico: Fendler, A. 1024 Isolectotype by Weatherby, C.A., J. Arnold Arbor. 25: 412.1944.

$=$ Selaginela rupestris $\mathrm{f}$. bourgeaui (Hieron) Clute.

=Lycopodium rupestre L. Species Plantarum 2:1101. 1753

=Selaginella bourgeaui Hieron.

Herbácea postrada y algunas veces erecta sobre suelo o rocas; tallos radialmente simétricos, largos o moderadamente cortos, trepadora a decumbente; rizóforos acomodados en la parte superior de los tallos o a todo lo largo del tallo; hojas monomórficas, apresas de manera compacta, ascendente, verde y ocasionalmente rojiza, lineal o lineal-lanceolada. Nervaduras abaxiales bien definidas; base cuneada y decurrente sobre el envés a redondeadas y adnatas en el haz, pubescentes o glabras, márgenes con cilios largos, transparentes, dispersos, ápice ligeramente acuminado.

Observaciones: Crece en bosques, entre las rocas o sobre ellas, a más de 2000 msnm. Prospera en lugares sombreados y expuestos, prefiriendo los primeros. Colectada en países como Canadá, USA, México, Colombia (Chocó) (Missouri Botanical Garden - $w^{3}$ - Specimen Data Base - 2007).

Material examinado: COLOMBIA. Chocó; Quibdó: Corr. Pacurita, bp-T, Palacios, L. 185

Selaginella speciosa A_Braun. Ann Sci Nat Bot. ser. 5 (3): 273.1865.

Tipo: Colombia, Bogotá, Triana sn (Isótipo: B, BM) 
$=$ Selaginella huberi $\mathrm{H}$. Christ; Bull Herb Boissier. ser. 2,2,1:73(1901).

Planta terrestre, tallo erecto; rizóforos básales; hojas medias adpresas o bastante unidas, lanceoladas, ovobadas, margen entero a ciliado; hojas laterales lanceoladas, ápices acuminados, agudos; hojas axilares elípticas, margen entero, ápice aristado.

Observaciones: Presente en zonas húmedas, de alta montaña, zonas intervenidas, con registro para los países de Colombia (Antioquia, Chocó), Ecuador y Perú. Desde los 01500 msnm (Missouri Botanical Garden - $w^{3}$ - Specimen Data Base - 2007).

Material examinado: COLOMBIA. Chocó; Bajo Baudó: Puerto Pizano, R. Baudó, $2 \mathrm{~km} \mathrm{~N}$, playa estero José Ángel, 300 m, Fuchs, H.P 22262; Snía. del Baudó, Pie de Pató, Espina, J. 11685. Paimadó: carr. Panamericana, R. Pató, 200 m, Forero, E 5426; Jequedó, $48 \mathrm{~km}$ W de Las Ánimas, R. Pasó, $250 \mathrm{~m}$, Gentry, A.H24040. Quibdó: Carr. -Tutunendó-San Francisco de Ichó, R. Ichó, Chaparraido, 100 m, Santa, J. 1163. Itsmina: Alto San Juan, Est. Cerr. Primera Mojarra, el Soldado, Espina, J. et al. 1355; GIPN 418. San José del Palmar: can-. Nóvita, cnías. Curundó, 250 m, Forero, E 3526.

Selaginella tomentosa Spring. Bull Mem Acad Royal Sci. Belgique 24:231.1849.

Tipo: Perú. Amazonas, Brent Berlin 633 (Isótipo: Imagen digital MO!)

Planta terrestre, postrada, tallo reptante, erecto; rizóforos basales; hojas medias elípticas, ovadas, margen ciliado, serrulado, ápice aristado; hojas laterales lanceoladas, base truncada, margen entero, ápice truncado, agudo.

Observaciones: Especie de zonas húmedas, intervenidas. Reportada para países como Colombia (Antioquia, Chocó), Ecuadory Perú. Desde los 150-1000 msnm. (MissouriBotanical Garden - $w^{3}$ - Specimen Data Base - 2007).

Material examinado: Chocó; Bahía Solano: $06^{\circ} 21^{\prime} 00^{\prime \prime} \mathrm{N}$ 076²6’00"W J. Espina, F. García \& S. Pino 2679. Pie de Pato: Serranía del Baudó, Espina, J., F. García y L. A. Lemus 1685; Animas-Pie de Pato, ca. $4 \mathrm{~km}$ a la propiedad del Sr. Gutiérrez, Croat, T. B. 56114, 56613. ca. $48 \mathrm{~km} \mathrm{~W}$ of Las Animas on Pan American Highway (under construction). $A$. Gentry \& E. Renteria A. 24040. Itsmina: Primera Mojarra, GIPN 417. Quibdó: carr. Tutunendó-San Francisco de Ichó, R. Ichó, 50 m, Santa, J. 1159.

Selaginella willdenowii (Desv_ex Poir_) Baker. Gard Chron. 783,950. 1867.

Tipo: «India Orientalis». Designado por Proctor 1985, Anon. (P-Herb. Desv.). (Lectotipo)

$=$ Lycopodium willdenowii Desv. ex Poir._Encyclopédie Méthodique. Botanique. Supplément 3: 552. 1814.

Planta terrestre, tallos rojizos a verdes o pajizos, erectos, glabros, isofilos desde la base hasta la primera ramificación; rizóforos restringidos a la base del tallo principal; hojas medias serruladas, a ciliadas en la base, ovadas; hojas axilares auriculadas, rojizas por el envés y por el haz verdoso; hojas laterales oblongas, base auriculada, ápice obtuso u agudo.

Observaciones: Especie originaria de Mayanmar, Malasia, Indonesia, Filipinas; introducida en los Estados Unidos, Panamá, Colombia (Antioquia, Chocó), Jamaica, Puerto Rico. En bosques húmedos, bordes de camino. Desde los 50-900 msnm(Missouri Botanical Garden - $w^{3}$ - Specimen Data Base - 2007).

Material examinado: Chocó; Quibdó: Barrio San Jose, Parte Alta, 50 msnm., Palacios, L. 184

\section{CONCLUSIONES}

Como agentes colonizadores de zonas intervenidas, las especies de Selaginella presentan una exuberante diversidad que se ostenta en los bosques húmedos y lluviosos tropicales como los de la región Chocó, trayendo consigo una mayor atención por parte de los investigadores, gracias a su valor biológico (taxonómico) y económico todavía desconocido.

Las características morfológicas tanto de los tallos como de las hojas dorsales, laterales y axilares son de gran importancia en la determinación de las especies incluidas en este trabajo.

Las especies más ampliamente distribuidas en el departamento del Chocó son: Selaginella articulata, S. atirrensis, $S$. exaltata, S. geniculata, S. haematodes, S. longissima y $S$. oaxacana.

\section{AGRADECIMIENTOS}

A la Asociación Nacional de Estudiantes de Ciencias Biológicas (ANECB) Seccional Chocó, a los biólogos Jhon Arley Palacios, Wilber Pino Chala, Anilio Castro Pérez, al igual que a la profesora María Teresa Murillo del Instituto de Ciencias Naturales por sus sugerencias y comentarios sobre el manuscrito.

\section{LITERATURA CITADA}

Alston A. H. G., Jermy, A. C. Y Rankin, J. M. 1981. Selaginella P. Beauv. Bull Brit Mus (Nat Hist) Bot. 9: 233-303.

Alston, A. H. G. 1955. The Heterophyllous Selaginella of Continental North America. Bull Brit Mus (Nat Hist) Bot.1 (8): 219-74.

Baker, J. G. 1883. A synopsis of the genus Selaginella, pt. 1. J Bot 21: 1-5. Corporación Autónoma para el Desarrollo Sostenible del Chocó(CODECHOCÓ). 1997. Plan de gestión ambiental urbano. Versión preliminar. Quibdó: Editorial de Autores Chocoanos. 33 pp.

Espinal, T. 1977. Zonas de vida o formaciones vegetales de Colombia. Memorias explicativas sobre el mapa ecológico. Vol. XIII, N II. Bogotá: IGAC. 238 pp.

Forero, E., A. Gentry. 1989. Lista anotada de las plantas del departamento del 


\section{Bioetnia Volumen 5 No 2 (julio-diciembre), 2008}

Chocó-Colombia. Bogotá: Biblioteca José Jerónimo Triana. Instituto de Ciencias Naturales-MHN. Universidad Nacional de Colombia.142 pp.. Gregory, D., Riba, R. 1979. Selaginella P. Beauv. Fl. Veracruz 6:1-35. Jermy, A. C. 1986. Subgeneric names in Selaginella. Fern Gaz. 13: 117-8. Murillo-A., J. 2004. Catálogo de pteridofitos en el Chocó biogeográfico. En: Rangel, O. (ed.). Diversidad biótica IV: El Chocó biogeográfico/Costa pacifica. Bogotá: Unilibros. p. 517-58.

Méndez C. M., Sarmiento J., Murillo A. 2002. Composición y diversidad de los Pteridofitos de Santa María (Boyacá, Colombia). En: Rangel - Ch., J. O., J. Aguirre-C., M.G. Andrade-C. (eds.) Libro de resúmenes Octavo Congreso Latinoamericano y Segundo Colombiana de Botánica. Bogotá: Editorial Unibiblios. p. 359.
Prada, C. 2004. Helechos. Capítulo 12. En: Izco, J. (Ed). Botánica. $2^{\mathrm{a} e d .}$ Editorial McGraw-Hill/Interamericana de España, S.A. (Aravaca). P. 143189

Rodríguez, D. W. 2002. Helechos, licopodios, selaginellas y equisetos del Parque Regional Arví. Medellín: CORANTIOQUIA-Jardín Botánico Joaquín Antonio Uribe. $260 \mathrm{pp}$.

Triana-M, Murillo-A. 2005. Helechos y plantas afines de Albán (Cundinamarca): El bosque subandino y su diversidad. Bogotá: Instituto de Ciencias Naturales-Universidad Nacional de Colombia, Instituto de Investigaciones de Recursos Biológicos «Alexander von Humboldt».162 pp.

Valdespino, I. 1993. Selaginella. In: Flora of North America. Vol. 2. Oxford University Press. Online at EFloras.org. Brittonia. 45: 315-27.

W3 Tropicos/Missouri Botanical Garden. Specimen Data Base. 2007. Available for Internet URL: http://mobot.mobot.org/W3T/vast.html 